15 Walden AP, Clarke GM, McKechnie S, et al. Patients with community acquired pneumonia admitted to European intensive care units: an epidemiological survey of the GenOSept cohort. Crit Care 2014; 18: R58.

16 Mannu GS, Loke YK, Curtain JP, et al. Prognosis of multi-lobar pneumonia in community-acquired pneumonia: a systematic review and meta-analysis. Eur J Intern Med 2013; 24: 857-863.

17 Claessens YE, Debray MP, Tubach F, et al. Early chest computed tomography scan to assist diagnosis and guide treatment decision for suspected community-acquired pneumonia. Am J Respir Crit Care Med 2015; 192: 974-982.

\title{
Implementing tuberculosis entry screening for asylum seekers: the Groningen experience
}

\author{
To the Editor:
}

For 3 years, Europe has faced an increasing refugee crisis in which hundreds of thousands of displaced persons are risking their lives to seek a safer and better future in the European Union (EU). Many of these migrants originate from highly tuberculosis (TB)-endemic countries. Furthermore, their travel conditions are often poor, allowing potential transmission of infectious diseases, including TB. The Netherlands have observed an increased influx of asylum seekers since 2012 too, with fluctuating numbers every month, and a sharp increase in the summer of 2015, putting constraints on the process of registration, identification, application and accommodation, including mandatory radiographic screening for intrathoracic TB. We describe the impact of such migration on the Public Health TB Clinic of Groningen (responsible for nearly all TB entry screening for asylum seekers in the Netherlands), and our flexible and efficient practice model for daily radiographic TB screening. We report the yield of this intervention over the last 4 years, and reflect on issues concerning entry screening.

In the Netherlands, a centralised system of asylum application is in operation. Apart from a small minority at the national airport, Schiphol (Amsterdam), and unaccompanied minors, the majority of asylum seekers must file their request at the national reception centre in Ter Apel, a former military North Atlantic Treaty Organization base, where they are received under basic conditions in prefabricated units in one of the hangars. The procedure of 1) registration and identity verification by the Alien Police, 2) mandatory radiographic screening for intrathoracic $\mathrm{TB}$, and 3) an interview with the Immigration and Naturalisation Service must be completed within a time frame of $72 \mathrm{~h}$.

Since April 2012, TB screening has been performed on a daily basis, including weekends, with a mobile digital X-ray unit parked in another hangar. Because of the increasing influx in 2014 and 2015, two annex reception centres were opened in the towns of Veenhuizen and Budel, the first one also being served by the Public Health TB Clinic of Groningen. Chest radiographs are immediately digitally transferred for reading to the Public Health TB Clinic in Groningen, approximately $50 \mathrm{~km}$ away, allowing for teleradiology. The chest radiographs from Ter Apel are read on a daily basis by one of the two public health TB physicians working in the Public Health TB Clinic. The chest radiographs from Veenhuizen are read by one of the two consultant chest physicians working in the modern TB sanatorium Beatrixoord (part of the University Medical Center Groningen (UMCG)). This is one of two national tertiary referral and expertise TB centres in the Netherlands. On Saturdays and public holidays, a group of Dutch TB physicians reads the chest radiographs on a rotational basis.

At the national reception centre, the Public Health TB Clinic uses a caravan as an on-site basic TB clinic. Apart from making chest radiographs, upon request of the TB physician or chest physician, the team of medical technical assistants from the Public Health TB Clinic in Groningen can, daily, on location, 1) take standardised questionnaires with telephone translators, 2) collect sputum for bacteriology, 3) perform tuberculin skin testing or 4) isolate individuals with abnormal chest radiographs possibly compatible with active intrapulmonary TB in three other caravans (with any relatives), awaiting the results of sputum-smear microscopy and GeneXpert (Cepheid, Sunnyvale, CA, USA) examination. Individuals with a chest radiograph highly suggestive of infectious pulmonary TB may be referred directly to Beatrixoord, which is equipped with state-of-the-art isolation rooms for 20 persons in addition to 10 beds for noninfectious TB patients, where further isolation, examination and treatment is offered. In the absence of productive cough, a bronchoscopy is arranged in the Chest Clinic of the UMCG. Sputum and bronchial lavage smear-microscopy and direct PCR test for Mycobacterium tuberculosis 
complex results may be available within 1 day. Between the four readers, on-demand (e-mail) consultations can be performed on a daily basis, with the participation of UMCG consultant paediatrician-infectiologists and a UMCG consultant pulmonologist-infectiologist, to exchange specific expertise and advice on chest radiograph abnormalities compatible or not compatible with intrathoracic TB.

The average daily number of screenings increased from 22 in 2012, to 34 in 2013, 67 in 2014 and 108 in 2015, with outliers above 300 persons per day in 2014 and 2015. Of all, nearly 75000 entrants, on average $1.8 \%$ per year, were further examined with structured questionnaires, additional chest radiographs, sputum examination or tuberculin skin tests, of which, on average, $4.1 \%$ were diagnosed with TB (table 1). Over almost 4 years, entry screening identified 55 TB cases, of which 18 (33\%) were smear positive, 48 (87\%) culture positive and six (11\%) were multidrug resistant. The annual prevalence of entry screening was 140 , 65, 65 and 75 cases per 100000 persons, respectively.

After the screening process, $98 \%$ of the asylum seekers with an unremarkable or acceptable chest radiograph, as well as the majority of the persons with radiographic abnormalities, could be transferred to a residential centre for asylum seekers elsewhere in the Netherlands. For a minority, follow-up examinations at regional public health TB clinics, general chest physicians or other medical specialists in general or university hospitals were arranged. For the majority of these cases, this decision could be based upon the available information at a distance and without transporting the person to the public health TB clinic. The information from the standardised questionnaire, including the radiology report and, when performed, available bacteriology results, were used for the referral letter. After a diagnosis of noninfectious TB treatment under Directly Observed Treatment is given in residential centres for asylum seekers, supervised by the regional public health TB clinic.

Apart from the logistics, another challenge is maintaining good standards of medical practice [1] under pressure of timely processing of the asylum application procedure with a variable influx of individuals, while at the same time, avoiding overdiagnosis and unnecessary further examinations. The Groningen experience shows that when screening asylum seekers (or other high-risk groups), reading of chest radiographs by a limited number of experienced, high-volume readers is advantageous, absorbing the fluctuating numbers of chest radiographs without too many problems and allowing for uniform practices and procedures, and close consultation, thus yielding a high diagnostic accuracy and resulting in a small proportion of follow-up medical interventions [2]. Our experience further underlines the advantage of cooperation between public health and clinical workers.

Screening is "triage" and not clinical radiology of symptomatic individuals. As in breast and cervical cancer screening, a balance between too many false-positive and some false-negative results should be taken into account. Only $1.8 \%$ of nearly 75000 asylum seekers were further examined at entry and often, the structured questionnaire, with or without public health clinical consultation, provided sufficient information for an adequate decision and advice for follow-up. The high proportion of culture-positive TB patients found reflects maximum efforts for culture confirmation but might also indicate we have underdiagnosed some cases. The number of TB cases found actively and passively after entrance will be further researched as quality assessment of entry screening.

TABLE 1 Asylum seekers screened for tuberculosis (TB) in Ter Apel, the Netherlands, in 2012-2014

\begin{tabular}{|c|c|c|c|c|}
\hline Indicators per year & 2012 & 2013 & 2014 & $2015^{\#}$ \\
\hline Asylum seekers screened & 7869 & 12357 & 24627 & 29473 \\
\hline $\begin{array}{l}\text { Persons with abnormal chest radiography and follow-up } \\
\text { examinations ( } \% \text { of all entrants) }\end{array}$ & $218(2.8)$ & $214(1.7)$ & $359(1.5)$ & $555(1.8)$ \\
\hline Structured questionnaire & 218 & 214 & 359 & 555 \\
\hline Additional chest radiography & 49 & 22 & 125 & 156 \\
\hline Sputum examination & 158 & 136 & 66 & 122 \\
\hline Tuberculin skin test & 17 & 10 & 19 & 21 \\
\hline TB cases diagnosed (\% of follow-up examinations) & $9(4.1)$ & $8(3.7)$ & $16(4.5)$ & $22(4.0)$ \\
\hline TB prevalence rate of entry screening per 100000 & 140 & 65 & 65 & 75 \\
\hline Pulmonary TB (\% culture positive) & $7(100)$ & $6(100)$ & $11(91)$ & 19 (95) \\
\hline Sputum or bronchial lavage smear positive & 2 & 2 & 4 & 10 \\
\hline Extrapulmonary TB (\% culture positive) & $2(50)$ & $2(50)$ & $5(60)^{+}$ & $3(67)$ \\
\hline Multidrug-resistant TB & 2 & 1 & 1 & 2 \\
\hline
\end{tabular}

Data are presented as $n$ unless otherwise stated. " : up to September 30; ๆ: auramine-stained microscopy, direct PCR and liquid culture; ${ }^{+}$: two cases of primary hilar TB in children without bacteriological examinations. 
Since 2001, TB incidence has been falling at an average rate of $4.3 \%$ per year in the World Health Organization European region but despite this notable progress in the past decade, TB is still a public health concern [3]. In many low-incidence countries, trends in TB incidence are driven largely by international migration dynamics. The special needs of migrants and cross-border issues are priority action areas in TB elimination [4], including continuity of care $[5,6]$. The yield of immigrant TB disease screening in the EU is several-fold higher than the TB incidence rates in low-incidence countries and even twice as high for asylum seekers [5]. The coverage of screening was highest at reception and residential centres. Similar results were later reported in Norway [7]. Chest radiography is the most sensitive screening instrument for intrathoracic TB among asylum seekers, compared to individual assessment of geographic origin, personal history and symptoms, also reducing delay between screening and start of treatment, reducing transmission and secondary cases [8]. We have used the structured questionnaire not to select asylum seekers eligible for chest radiography but to further investigate individuals with abnormal chest radiographs, and to reduce false-positive diagnoses and unnecessary examinations.

An evaluation of asylum seekers entry screening in the Netherlands over the past 5 years found a low yield among Syrian refugees, currently a dominating asylum-seeking group, and other asylum seekers from countries with a TB incidence of $<50$ cases per 100000 population [9]. In September 2015, the Minister of Health in the Netherlands suspended entry screening for asylum seekers from Syria. In the context of TB elimination, a next step would be to screen asylum seekers for latent TB infection (LTBI), and to offer preventive treatment to those testing positive with the tuberculin skin test and/or interferon- $\gamma$ release assays $[10,11]$. The effectiveness and cost-effectiveness of asylum seeker entry screening strategies are subject to debate [12-14], although targeting LTBI screening at asylum seekers at the highest risk of developing TB disease (e.g. those from high-incidence countries) might improve cost-effectiveness [5, 15], but further studies should be performed in the EU as well.

We describe a unique, flexible and efficient practice model for daily radiographic, early-diagnosis TB screening of a varying influx of asylum seekers that has proved feasible. Clearly, the Netherlands is a relatively small and well-organised country, but we believe we offer potential learning points for other European countries.

0 @ERSpublications

The Dutch practice model for radiographic TB screening of asylum seekers has proved feasible http://ow.ly/Z1QTP

Onno W. Akkerman ${ }^{1,2}$, Wiel C.M. de Lange ${ }^{1,2}$, Elisabeth H. Schölvinck ${ }^{3}$, Bert Wolters ${ }^{4}$, Yvonne Aartsma ${ }^{4}$, Tjip S. van der Werf ${ }^{2,5}$ and Rob van Hest ${ }^{4,6}$

${ }^{1}$ University of Groningen, University Medical Center Groningen, Tuberculosis Center Beatrixoord, Haren, The

Netherlands. ${ }^{2}$ University of Groningen, University Medical Center Groningen, Dept of Pulmonary Diseases and

Tuberculosis, Groningen, The Netherlands. ${ }^{3}$ University of Groningen, University Medical Center Groningen, Dept of Pediatrics, Groningen, The Netherlands. ${ }^{4}$ Regional Public Health Service Groningen, Dept of Tuberculosis Control, Groningen, The Netherlands. ${ }^{5}$ University of Groningen, University Medical Center Groningen, Dept of Internal Medicine, Groningen, The Netherlands. ${ }^{6}$ Regional Public Health Service Rotterdam-Rijnmond, Dept of Tuberculosis Control, Rotterdam, The Netherlands.

Correspondence: Onno W. Akkerman, University Medical Center Groningen, Tuberculosis Center Beatrixoord, Dilgtweg 5, 9750 RA Haren, The Netherlands. E-mail: o.w.akkerman@umcg.nl

Received: Jan 152016 | Accepted after revision: Feb 262016 | First published online: March 232016

Conflict of interest: None declared.

\section{References}

1 Migliori GB, Zellweger JP, Abubakar I, et al. European union standards for tuberculosis care. Eur Respir J 2012; 39: $807-819$.

2 Abubakar I, Story A, Lipman M, et al. Diagnostic accuracy of digital chest radiography for pulmonary tuberculosis in a UK urban population. Eur Respir J 2010; 35: 689-692.

3 European Centre for Disease Prevention and Control, WHO Regional Office for Europe. Tuberculosis Surveillance and Monitoring in Europe 2015. Stockholm, European Centre for Disease Prevention and Control, 2015.

4 Lönnroth K, Migliori GB, Abubakar I, et al. Towards tuberculosis elimination: an action framework for low-incidence countries. Eur Respir J 2015; 45: 928-952.

5 Klinkenberg E, Manissero D, Semenza JC, et al. Migrant tuberculosis screening in the EU/EEA: yield, coverage and limitations. Eur Respir J 2009; 34: 1180-1189.

6 Dara M, de Colombani P, Petrova-Benedict R, et al. The minimum package for cross-border TB control and care in the WHO European Region: a Wolfheze Consensus Statement. Eur Respir J 2012; 40: 1081-1090.

7 Harsted I, Jacobsen GW, Heldal E, et al. The role of entry screening in case finding of tuberculosis among asylum seekers in Norway. BMC Public Health 2010; 10: 670.

8 Schneeberger Geisler S, Helbling P, Zellweger JP, et al. Screening for tuberculosis in asylum seekers: comparison of chest radiography with an interview-based system. Int J Tuberc Lung Dis 2010; 14: 1388-1394. 
9 De Vries G, van Rest J, Meijer W, et al. Low yield of screening asylum seekers from countries with a tuberculosis incidence of $<50$ per 100000 population. Eur Respir J 2016; 47: 1870-1872.

10 Getahun H, Matteelli A, Abubakar I, et al. Management of latent Mycobacterium tuberculosis infection: WHO guidelines for low tuberculosis burden countries. Eur Respir J 2015; 46: 1563-1576.

11 Erkens CG, Slump E, Verhagen M, et al. Monitoring latent tuberculosis infection diagnosis and management in the Netherlands. Eur Respir J 2016; 47: 1492-1501.

12 Schwartzman K, Menzies D. Tuberculosis screening of immigrants to low-prevalence countries. A cost-effectiveness analysis. Am J Respir Crit Care Med 2000; 161: 780-789.

13 Dasgupta K, Menzies D. Cost-effectiveness of tuberculosis control strategies among immigrants and refugees. Eur Respir J 2005; 25: 1107-1116.

14 Mandalakas AM, Menzies D. Is screening immigrants for latent tuberculosis cost-effective? Lancet Infect Dis 2011; 11: 418-419.

15 Wingate LT, Coleman MS, de la Motte Hurst C, et al. A cost-benefit analysis of a proposed overseas refugee latent tuberculosis infection screening and treatment program. BMC Public Health 2015; 15: 1201.

\title{
Prevalence and factors associated with diabetes mellitus among tuberculosis patients: a nationwide cohort
}

\author{
To the Editor:
}

The association between diabetes mellitus (DM) and tuberculosis (TB) has been a matter of study worldwide, since it is assumed that DM triples the risk of TB [1]. Recent studies have found discrepant prevalence of DM among TB patients, ranging from 5.3\% in Denmark [2] to $44 \%$ in India [3]. There is an urgent need to control both epidemics in order to achieve the World Health Organization (WHO) TB elimination goal [4]. To reach this goal, an integrated approach between TB elimination strategies and control of noncommunicable diseases that perpetuate the risk for TB is fundamental [5].

Portugal has an intermediate incidence rate of TB and an estimated prevalence of DM of 7.4\% (estimated underdiagnosed prevalence of 5.7\%) [6]. There are no official recommendations to actively screen for DM in TB patients (there is only a provisional recommendation from the WHO Collaborative Framework for Care and Control of Tuberculosis and Diabetes [7]). A previous systematic review estimated that if TB patients were screened for DM, the DM prevalence would range widely, from $1.9 \%$ to $35 \%$ [8]. This study aims to assess DM prevalence among the Portuguese TB population and to identify which factors are associated with it. This is a Portuguese nationwide retrospective cohort study of a 6-year period (2008-2013).

Data were collected from the national TB database, SVIG-TB (Sistema de Vigilância da TB em Portugal (System for Surveillance of TB in Portugal)) [9]. All patients diagnosed with TB in Portugal are mandatorily recorded through this registry. We included all patients with newly diagnosed pleural-pulmonary TB who finished treatment between January 2008 and December 2013. Exclusion criteria were age $<18$ years, previous TB diagnosis, isolated extrapulmonary TB, multidrug- or extensively drug-resistant $\mathrm{TB}$ and unknown outcome (abandonment/emigration).

The following definitions were used. TB diagnosis: positive culture or both smear and nucleic acid amplification test positivity in a pleural or respiratory specimen; DM diagnosis: self-reported by the patient and/or based on clinical data; TB compliance: $\geqslant 80 \%$ of prescribed treatment; unsuccessful treatment: death, lack of microbiological conversion and/or incomplete treatment $(<80 \%$ of prescribed treatment); drug consumer: regular consumer of illicit drugs. All the comorbidities analysed (including DM itself) were reported to the Portuguese surveillance system, SVIG-TB [9].

For the statistical analysis, categorical variables were described by absolute (relative) frequencies, while continuous variables were described by the median (interquartile range (IQR)). For all variables, a comparative analysis between patients with and without DM was performed: Chi-squared or Fisher test (as adequate) for the study of independence among categorical variables, and the Mann-Whitney test for the assessment of statistically significant differences between two independent continuous variables.

DM prevalence $(6.0 \%)$ and the number of possible predictors hindered a straightforward application of a multiple logistic regression model. Initially, random oversampling of the DM class, until the two classes had 